\title{
High Sensitivity Temperature Sensor Based on Singlemode-no- Core- Singlemode Fibre Structure and Alcohol
}

\author{
Ke Tiana \\ Harbin Engineering University \\ Gerald Farrell \\ Technological University Dublin, gerald.farrell@tudublin.ie \\ Xianfan Wang \\ Harbin Engineering University
}

See next page for additional authors

Follow this and additional works at: https://arrow.tudublin.ie/engschmanart

Part of the Physics Commons

\section{Recommended Citation}

Wang, P. et al (2018) High Sensitivity Temperature Sensor Based on Singlemode-no-Core- Singlemode Fibre Structure and Alcohol, Sensors and Actuators A Physical. October 2018. doi: 10.1016/ j.sna.2018.10.016

This Article is brought to you for free and open access by the School of Manufacturing and Design Engineering at ARROW@TU Dublin. It has been accepted for inclusion in Articles by an authorized administrator of ARROW@TU Dublin. For more information, please contact arrow.admin@tudublin.ie, aisling.coyne@tudublin.ie, gerard.connolly@tudublin.ie.

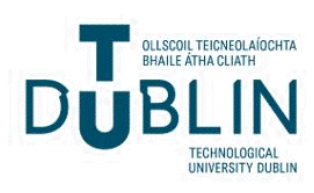




\section{Authors}

Ke Tiana, Gerald Farrell, Xianfan Wang, Yifan Xin, Yanqiu Du, Wenlei Yang, Haidong Liang, Elfed Lewis, and Pengfei Wang 


\title{
High sensitivity temperature sensor based on singlemode-no-core-singlemode fibre structure and alcohol
}

\author{
Ke Tian ${ }^{\mathrm{a}}$, Gerald Farrell ${ }^{\mathrm{b}}$, Xianfan Wang ${ }^{\mathrm{a}}$, Yifan Xin ${ }^{\mathrm{a}}$, Yanqiu Du ${ }^{\mathrm{a}}$, Wenlei Yang ${ }^{\mathrm{a}}$, Haidong Liang ${ }^{\mathrm{a}, \mathrm{d}}$, Elfed Lewis ${ }^{\mathrm{c}}$, \\ Pengfei Wang ${ }^{\mathrm{a}, \mathrm{e}, *}$ \\ ${ }^{\mathrm{a}}$ Key Laboratory of In-fiber Integrated Optics of Ministry of Education, College of Science, Harbin Engineering University, Harbin 150001, China \\ b Photonics Research Centre, Dublin Institute of Technology, Kevin Street, Dublin 8, Ireland \\ c Optical Fibre Sensors Research Centre, Department of Electronic and Computer Engineering, University of Limerick, Limerick, Ireland \\ d Hands and Feet Microsurgery, the Second Hospital of Dalian Medical University, Dalian 116023, China \\ ${ }^{\mathrm{e}}$ Key Laboratory of Optoelectronic Devices and Systems of Ministry of Education and Guangdong Province, College of Optoelectronic Engineering, Shenzhen University \\ Shenzhen, 518060, China
}

\section{A R T I C L E I N F O}

\section{Article history:}

Received 28 April 2018

Received in revised form 21 September 2018

Accepted 10 October 2018

Available online $\mathrm{xxx}$

Keywords:

Optical fibre sensor

Multimode interference

Alcohol

Temperature sensing

\section{A B S T R A C T}

A high sensitivity temperature sensor based on a singlemode-no-core-singlemode (SNCS) fibre structure and surrounded with alcohol within a silica capillary is described. In this investigation, no-core fibre (NCF) is used as the multimode waveguide and alcohol is chosen as the temperature sensitive medium. By packaging the alcohol solution with a short length of NCF enclosed within a silica capillary, the surrounding temperature can be detected by monitoring the variations of transmission loss at a specific wavelength. The theoretical analysis predicts this temperature sensor can provide high sensitivity, and the experimental results support this. The maximum temperature sensitivity of the sample is $0.49 \mathrm{~dB} /{ }^{\circ} \mathrm{C}$ with a potential temperature resolution of 0.02 ${ }^{\circ} \mathrm{C}$ at the operating wavelength of $1545.9 \mathrm{~nm}$. In addition, the repeatability and response time of the sensor of this investigation are investigated experimentally.

(C) 2018

\section{Introduction}

In recent years, singlemode-multimode-singlemode (SMS) fibre structures have attracted much research interest, primarily due to several advantages, including compact structure, ease of fabrication, and excellent compatibility with other photonic and optical fibre devices $[1,2]$. Relying on multimode interference (MMI) within the multimode waveguide and the resulting self-imaging phenomenon, SMS fibre structures have been widely applied in telecommunications, for example as wavelength division multiplexers [3], edge filters [4] and bandpass filters [5]. In addition, SMS fibre structures have also been successfully developed as optical fibre sensors for a wide variety of measurements, e.g. temperature [6], strain [7], curvature [8], humidity [9], refractive index (RI) [10] and magnetic fields [11,12]. SMS fibre structures can be easily adapted for a wide range of different applications due to the versatility of their fabrication and the fact that all versions (variants) rely on a common underlying operation principle i.e. MMI that exists within the multimode fibre (MMF).

Considerable research effort has been directed towards accurate temperature measurement over a long period as it is considered an im-

\footnotetext{
* Corresponding author at: Key Laboratory of In-fiber Integrated Optics of Ministry of Education, College of Science, Harbin Engineering University, Harbin 150001, China. Email address: pengfei.wang@dit.ie (P. Wang)
}

portant physical parameter in many fields, and consequently, a wide variety of temperature sensors have been developed to date. When compared with traditional electronic temperature sensors e.g. thermocouples and thermistors, optical fibre based sensors exhibit a range of unique advantages including compact size, stability in hostile environments, inherent immunity to external electromagnetic interference, and excellent potential to offer high accuracy measurement (including high sensitivity) which is required for applications that involve to detecting very small temperature changes, for example in biological and medical diagnostics. Optical fibre temperature sensors have been predominantly based on fibre grating structures including fibre Bragg grating (FBG) [13] and long period grating (LPG) [14]. However, fibre grating structures generally offer a relatively low temperature sensitivity of circa $10 \mathrm{pm} /{ }^{\circ} \mathrm{C}$. Various fibre interferometer based temperature sensors have become increasingly popular due to their excellent sensing performance, for example those based on the Mach-Zehnder interferometer (MZI) [15], the Fabry-Perot interferometer (FPI) [16], the Sagnac interferometer [17] and the Michelson interferometer [18]. However, in most cases, fibre interferometer based optical fibre sensors require a relatively complicated fabrication process which increases potential fabrication/manufacturing cost. In recent years, MMI based temperature sensors have attracted much research attention due to the advantages of potentially low raw material cost and ease of fabrication compared with other fibre optic sensing configurations. SMS fibre structures can be used in temperature measurement and this arises from the well-known thermo-optic and thermal expan- 
sion effects which are intrinsic material properties of silica optical fibre. Being dependent on this property, surrounding temperature changes produce a significant influence on MMI within MMF section, and monitoring the resulting spectral shift or intensity variation has been widely applied as an interrogation method in many reported temperature sensing scenarios [19-23]. However, SMS fibre structures based temperature sensors have often yielded relatively low intensity-temperature sensitivities (reported as optical transmission intensity per unit temperature) of about $0.0007 \mathrm{~dB} /{ }^{\circ} \mathrm{C}[21], 0.0508 \mathrm{~dB} /{ }^{\circ} \mathrm{C}$ [22] and $0.063 \mathrm{~dB} /{ }^{\circ} \mathrm{C}$ [23], which can be attributed to the inherently low thermo-optic coefficients of the silica material. Thus, there is a clear need to develop a temperature sensor based on an SMS fibre structure that is easily fabricated, low-cost and which can offer higher measurement sensitivity.

In this paper, an optical fibre temperature sensor is described based on a singlemode-no-core-singlemode (SNCS) fibre structure surrounded by an alcohol layer. Alcohol was chosen as the temperature sensitive material to improve the sensing performance of the sensor due to its relatively high thermos-optic coefficient. The simulation analysis predicts this compact and simple structure can provide high sensitivity in terms of optical transmission intensity per unit temperature. The experiments undertaken as part of this investigation further support the existence of high sensitivity in this device. This indicates its potential applications in accurate bio-temperature monitoring as well as many other environment monitoring fields.

\section{Principle and theoretical simulation}

The schematic configuration of the proposed temperature sensor is shown in Fig. 1, an SNCS fibre structure is surrounded by alcohol solution within a silica capillary tube, and the two ends of the capillary tube were sealed using ultraviolet (UV) glue. In this investigation, the alcohol-immersed SNCS fibre structure is a generic SMS structure, in which the no-core fibre (NCF) is coupled between two optically aligned single-mode fibres (SMFs), and the NCF section and the surrounding sealed alcohol solution represent the multimode waveguide core and the associated cladding, respectively. It is well known that in an SMS fibre structure, the launched light from the input SMF can be assumed to be Gaussian with a radial field distribution of $E(r, 0)$ on account of the input SMF's circular symmetry and the fact that it supports transmission of only the fundamental mode. When the transmission light is launched into the NCF section, a series of high-order modes are excited and the input field can be decomposed into the eigenmodes of the linearly polarized (LP) mode of $\mathrm{LP}_{\mathrm{nm}}$. However, in this case only the $\mathrm{LP}_{0 \mathrm{~m}}$ modes can be effectively excited due to the circular symmetry of the input field and the assumption of ideal alignment between the SMF and NCF. For an alcohol-immersed NCF, the excited mode number $M$ of the $\mathrm{LP}_{0 \mathrm{~m}}$ can be calculated using:

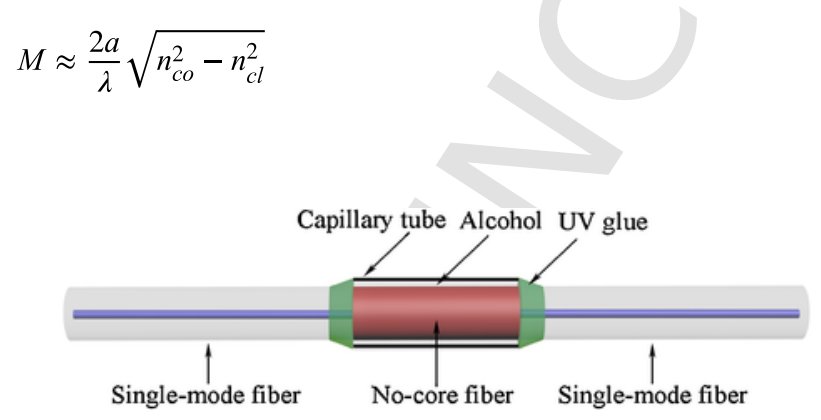

Fig. 1. Schematic of the sensing head based on an alcohol-immersed SNCS fibre structure. where $a$ is the radius of the NCF core, $n_{c o}$ and $n_{c l}$ are the RI for the $\mathrm{NCF}$ core and the surrounding alcohol solution, respectively, and $\lambda$ is the free space wavelength. By denoting the field profile of $\mathrm{LP}_{0 \mathrm{~m}}$ as $\varphi_{m}(r)$, the input light field $E(r, 0)$ of the NCF can be expressed as [24]:

$$
E(r, 0)=\sum_{m=1}^{M} c_{m} \varphi_{m}(r)
$$

where $r$ is radial distance in the cross section of the fibre, $c_{m}$ is the excitation coefficient corresponding to the $\mathrm{LP}_{0 \mathrm{~m}}$ mode, which can be calculated by the overlap integral between $E(r, 0)$ and $\varphi_{m}(r)$ [25]:

$$
c_{m}=\frac{\int_{0}^{\infty} E(r, 0) \varphi_{m}(r) r d r}{\int_{0}^{\infty} \varphi_{m}(r) \varphi_{m}(r) r d r}
$$

As the light propagates axially within the NCF section, the field at a propagation distance $\mathrm{z}$ can be calculated by [26]:

$$
E(r, z)=\sum_{m=1}^{M} c_{m} \varphi_{m}(r) \exp \left(i \beta_{m} z\right)
$$

where $\beta_{m}$ is the propagation constant of each eigenmode of the NCF. MMI occurs within the NCF section between these excited high-order modes. When the light arrives at the output SMF, a portion of light from the NCF is coupled into the core of the output SMF, while the remaining light signal is coupled into the cladding and excites cladding modes which decay with a short distance. Ultimately, the resultant output optical intensity can be expressed as [12]:

$$
I(\lambda)=\sum_{s=1}^{M} \eta_{s}^{2} \cdot I_{0}(\lambda)+\sum_{s \neq m=1}^{M} \eta_{s} \cdot \eta_{m} \cdot I_{0}(\lambda) \cdot \cos (2 \pi \Delta n L / \lambda)
$$

where $I_{0}$ is the intensity of the $\mathrm{LP}_{01}$ (fundamental mode) in the SMF, $\eta_{s}$ and $\eta_{m}$ is the coupling coefficient of the $\mathrm{LP}_{0 \mathrm{~s}}$ and the $\mathrm{LP}_{0 \mathrm{~m}}$ mode, respectively, $\Delta n$ is the effective refractive index difference between the two modes and $L$ is the length of the NCF. When the surrounding RI (outside the NCF) varies, the effective RIs of the different modes also changes, which influences the MMI and results in changes to the optical transmission intensity according to Eq. (5) above. Alcohol is chosen as the cladding material of the NCF because it is a widely available, can be easily infiltrated into the silica capillary of the device in this investigation and is a low-cost liquid but with a relatively high thermo-optic coefficient of $-4 \times 10^{-4} \mathrm{RIU} /{ }^{\circ} \mathrm{C}$ (a value confirmed in the experiments described below) which is two orders of magnitude higher than the equivalent value of $6.9 \times 10^{-6} \mathrm{RIU} /{ }^{\circ} \mathrm{C}$ for silica fibre [27]. Utilizing the linear relationship between the RI of the alcohol solution and temperature, the external temperature can be measured based on an SNCS fibre structure combined with alcohol. It is worth noting that the temperature induced thermal expansion of the alcohol solution causes an extra stress in the silica capillary, however in practice this additional stress is so small that it can be ignored.

Based on the above theoretical analyses, numerical simulations have been conducted employing the beam propagation method (BPM) prior to fabrication of the sensor. The simulation conditions 
were based on a mesh size in the $\mathrm{X}, \mathrm{Y}$ and $\mathrm{Z}$ directions of $0.1 \mu \mathrm{m}$, $0.1 \mu \mathrm{m}$ and $1 \mu \mathrm{m}$, respectively, and the boundary condition adopted a perfectly matched layer (PML) condition in the model. The length of $\mathrm{NCF}$ was chosen to be $4 \mathrm{~cm}$ which is an effective length to allow the occurrence of multimode interference (MMI) and this length is considered an optimal value to offer high temperature sensitivity [10,24]. The calculated optical field intensity distributions within the NCF section both before and after the injection of alcohol (the assumed RI value of the alcohol was 1.363 at $20^{\circ} \mathrm{C}$ ) at the wavelength of $1550 \mathrm{~nm}$ are presented in Figs. 2(a) and (b) respectively. The regular MMI pattern within the NCF section remains similar when the surrounding RI changes from 1 (the RI of air) to 1.363 . The corresponding simulated transmission spectrum over the wavelength range of $1500 \mathrm{~nm}$ to $1600 \mathrm{~nm}$ is presented in Fig. 2(c). From Fig. 2(c), it is clear that the resonant wavelength evolves from $\mathrm{dip}_{1}$ to $\mathrm{dip}_{2}$ accompanied by a clear wavelength red shift $(17.2 \mathrm{~nm})$ and intensity decrease $(3.58 \mathrm{~dB})$ after alcohol is injected into the capillary tube.

(a)

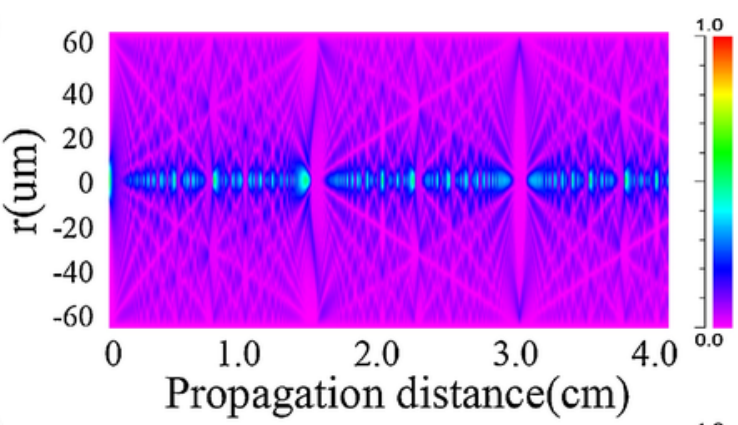

(b)

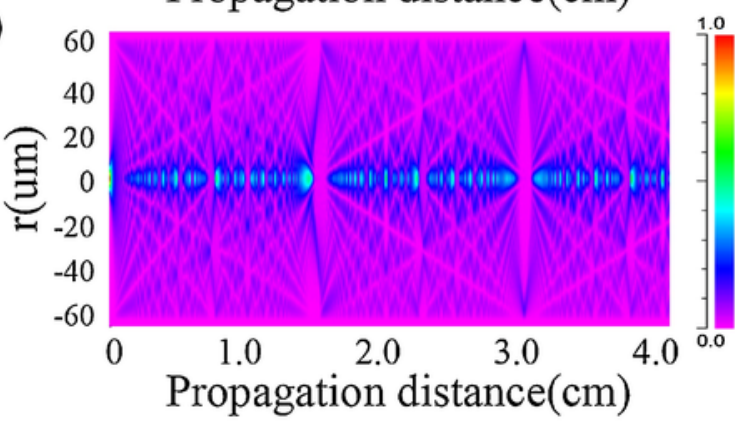

(c)

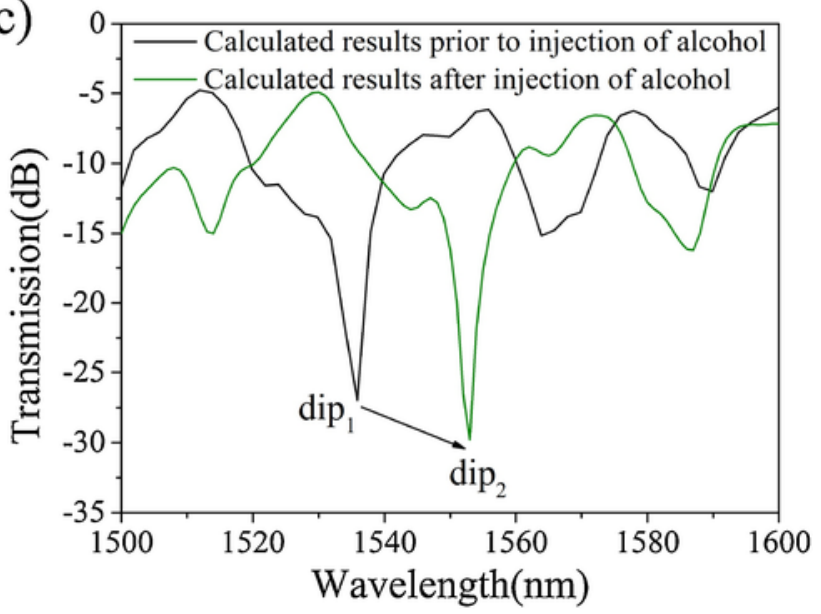

Fig. 2. Simulated optical field intensity distribution within an SNCS fibre structure (a) before injection of alcohol; (b) after injection of alcohol; (c) The corresponding transmission spectrum scanning from $1500 \mathrm{~nm}$ to $1600 \mathrm{~nm}$.
To underpin a theoretical prediction for the amplitude change of the single wavelength dip $_{2}$ located at $1553 \mathrm{~nm}$, it is necessary to know how the RI of the alcohol changes with temperature. For this purpose, the temperature dependence of the RI of the alcohol solution was experimentally measured and the results are shown in Fig. 3. It is clear that as the temperature increases, the RI of the alcohol solution exhibits a linear decrease (green line) with a slope value of temperature dependence circa $-0.0004 \mathrm{RIU} /{ }^{\circ} \mathrm{C}$ which is consistent well with the result reported in [28]. Using this linear relationship, the amplitude of the $1553 \mathrm{~nm}$ dip was calculated at different temperatures. The calculated results (pink stars in Fig. 3) show that the intensity (in $\mathrm{dB}$ ) of the $1553 \mathrm{~nm}$ dip is increased when the surrounding temperature increases from $20^{\circ} \mathrm{C}$ to $45^{\circ} \mathrm{C}$. The data was fitted using a polynomial (quadratic) function which yielded a high correlation (fitting) coefficient of 0.9996 . From the results, a maximum intensity-temperature sensitivity of $0.63 \mathrm{~dB} /{ }^{\circ} \mathrm{C}$ was achieved at $20^{\circ} \mathrm{C}$. These simulation results predict that it is possible to fabricate a practical temperature sensor based on MMI employing an SNCS fibre structure and alcohol solution. Furthermore, it also demonstrates the feasibility of an interrogation scheme based on intensity changes at a single operating wavelength.

\section{Experiments and discussion}

The NCF used in this experiment was a commercially available product (Thorlabs FG125LA) which is made of pure silica with a diameter of $125 \mathrm{um}$, with an RI value of 1.444. A section of NCF with a length of $4 \mathrm{~cm}$ was initially stripped, cleaved, and optically connected between two single-mode fibres (SMF-28) using a standard fusion splicer (Fujikura 62S) to construct the SNCS fibre structure. The SNCS fibre structure was placed in the center of a capillary tube, whose inner diameter was $600 \mu \mathrm{m}$ and length was $5 \mathrm{~cm}$. Next, the alcohol solution was injected into the capillary tube with the help of capillary force. The concentration of the alcohol used in the experiment was $98 \%$, and its RI was measured as 1.363 at $20^{\circ} \mathrm{C}$ using an Abbe refractometer. Finally, to complete the fabrication process, the two ends of the capillary tube were sealed using UV curable glue to avoid alcohol leakage and evaporation. The measured transmission spectrum of the fabricated SNCS fibre structure is shown in Fig. 4 (blue line). The red line in Fig. 4 represents the transmission spectrum of the SNCS fibre structure after injection of alcohol. From Fig. 4, it can be observed that after the injection of alcohol, the interference

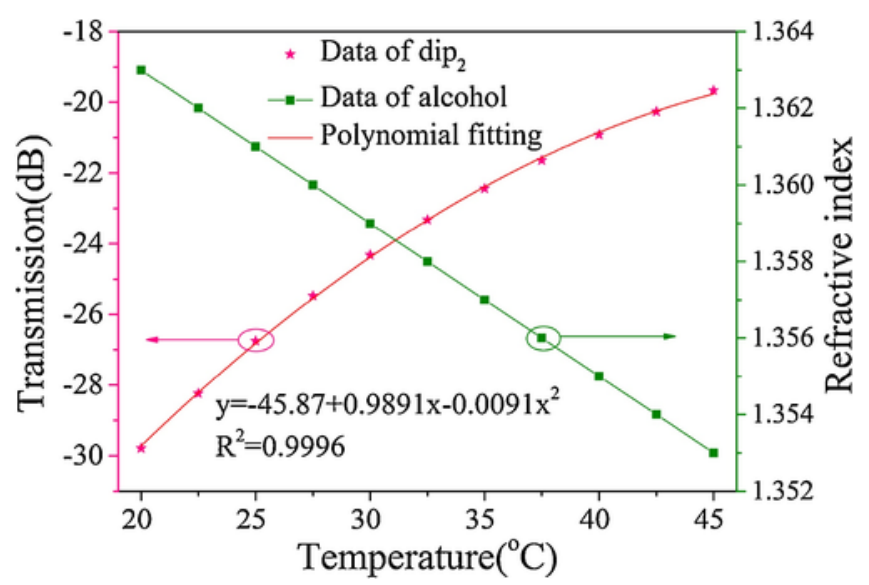

Fig. 3. Temperature dependence of refractive index of alcohol and simulated transmission loss (in $\mathrm{dB})$ at single wavelength $(1553 \mathrm{~nm})$ when surrounding temperature changed. 


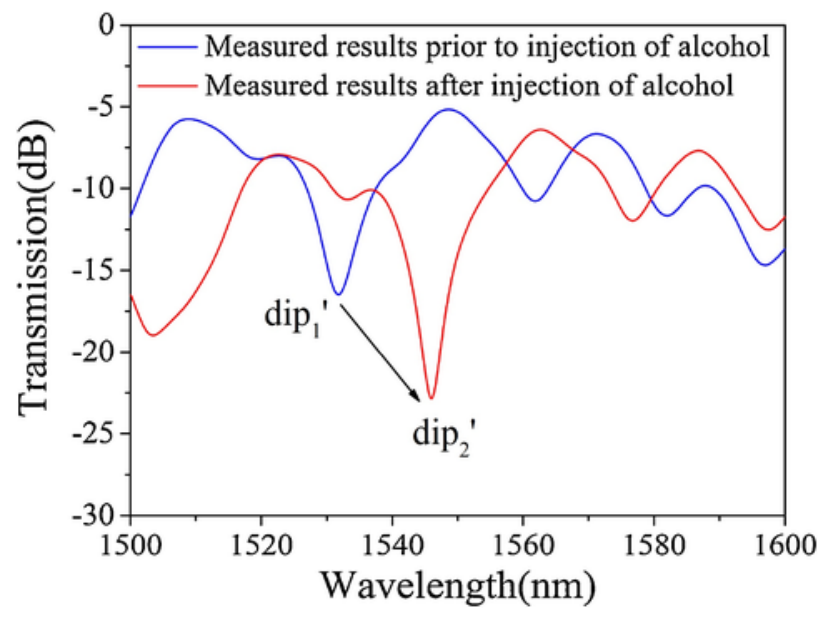

Fig. 4. Measured transmission spectra of the SNCS fibre structures before and after injection of alcohol.

$\operatorname{dip}_{1}^{\prime}$ moved to a longer wavelength (a change of $14.1 \mathrm{~nm}$ ). Also, the intensity of $\operatorname{dip}_{1}{ }^{\prime}$ exhibits a significant decline (increase in loss of $6.381 \mathrm{~dB}$ ). The observed dip evolution is consistent with the simulated results presented in Fig. 2(c). The discrepancy that exists between the simulated and measured results can be attributed to the fact that the meridional axes of the SMF and NCF are not exactly in ideal central alignment [24]. Also there are some inherent approximations in the BPM simulation, for example only $\mathrm{LP}_{0 \mathrm{~m}}$ modes were excited in the theoretical module. Furthermore, the solidification of the UV glue may also induce additional perturbation due to the microbend stress.

The experimental setup for temperature measurement using the proposed temperature sensor is schematically depicted in Fig. 5. The fabricated sample was placed on a temperature controlled thermoelectric Peltier cooler and fixed using UV glue to ensure that the sample can feed back surrounding temperature variation precisely and timely. A stable power supply (TTI EL302RT) was used to control the input electric current of the thermoelectric Peltier cooler, and the real-time temperature value on its surface was monitored using a thermocouple (RS 1313). The input SMF of the sample was connected to a broadband light source (BBS, Thorlabs S5FC1005S). The light was transmitted through the sensor and finally recorded by an optical spectrum analyzer (OSA, Agilent 86142B). The sample and the thermoelectric Peltier cooler were coved using a simple customized sealing (heat insulation) chamber to ensure more accurate temperature control in the experiments.

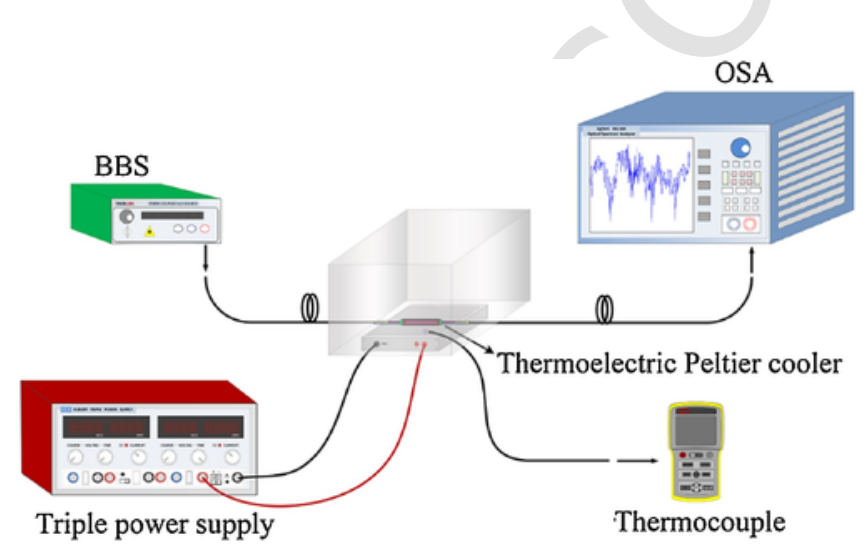

Fig. 5. Schematic diagram of the experimental setup for temperature measurement.
Fig. 6 shows the transmission spectrum evolution as the temperature was increased from $20^{\circ} \mathrm{C}$ to $45^{\circ} \mathrm{C}$ with an increment of $5^{\circ} \mathrm{C}$. From Fig. 6, it can be seen that the transmission loss (intensity) of the interference $\operatorname{dip}_{2}{ }^{\prime}$ (at the wavelength of $1545.9 \mathrm{~nm}$ ) exhibits an expected increase $(6.97 \mathrm{~dB})$ as the temperature was increased from $20^{\circ} \mathrm{C}$ to $45^{\circ} \mathrm{C}$. In addition, there is a small wavelength shift with the temperature increase, but this is not the focus of this investigation, as the sensor interrogation is planned to be purely intensity based. As in the case of the theoretical predictions, a clear relationship between temperature and transmission loss (in $\mathrm{dB}$ ) is established. The dip transmission intensity was plotted (red marks) and fitted (red line) against temperature in the range of $20^{\circ} \mathrm{C}$ to $45^{\circ} \mathrm{C}$ in Fig. 7 . The quadratic fitting curve exists a favorable relationship $\left(\mathrm{R}^{2}=0.9942\right)$ between the transmission intensity and temperature, and the maximum intensity-temperature sensitivity was $0.49 \mathrm{~dB} /{ }^{\circ} \mathrm{C}$ when the temperature was $20^{\circ} \mathrm{C}$. Furthermore, given the limiting $0.3 \mathrm{~dB}$ intensity resolution of the OSA used in the experiments, the temperature resolution of the sensor system was calculated to be $0.61{ }^{\circ} \mathrm{C}$. In practice the potential resolution achievable can be as good as $0.02 \mathrm{~dB}$, for example replacing the detection device of OSA by a standard optical power meter with a higher intensity measurement resolution of $0.01 \mathrm{~dB}$. Also a dedicated interrogation system with appropriate signal processing e.g. averaging should be applied to reduce the effect of noise.

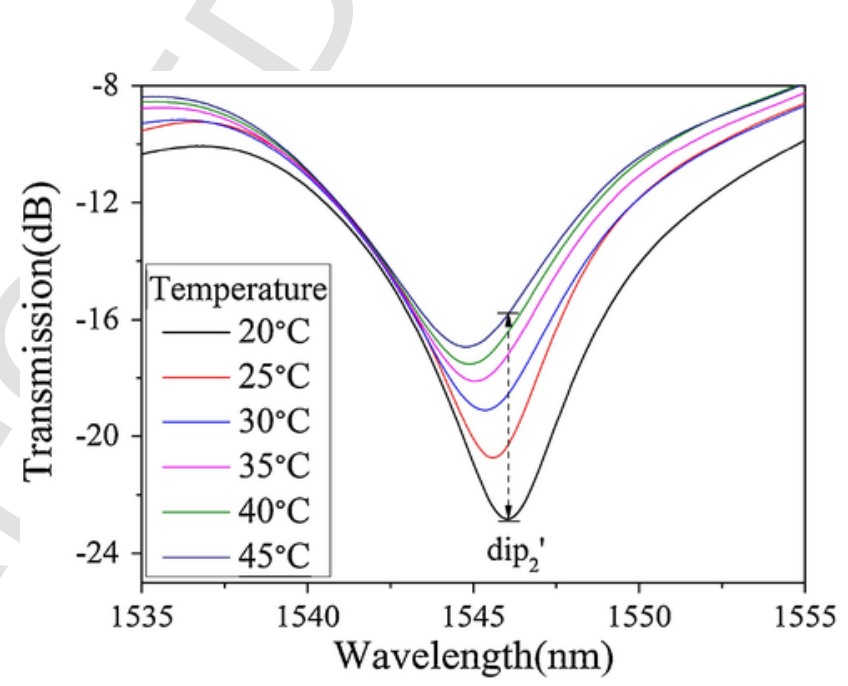

Fig. 6. Measured transmission spectrum evolution at varying temperature.

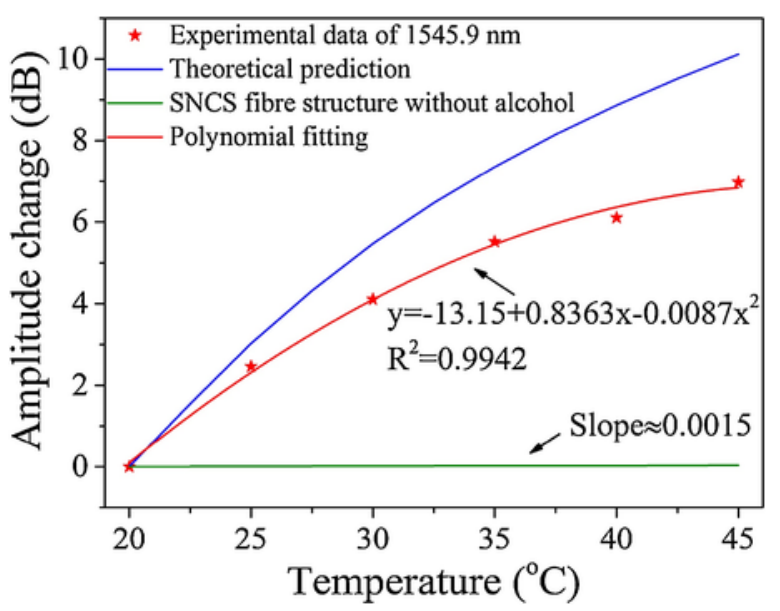

Fig. 7. Amplitude (transmission loss) changes as a function of temperature. 
Fig. 7 also includes a direct comparison between the experimental and theoretical results. As shown in Fig. 7, there exists a good agreement between the measured results and the theoretical predictions, in that both fitting curves exhibit similar trends with temperature variation. The discrepancy between the measured and simulated results can be attributed to: (1) the thermo-optic and thermal expansion effects of optical fibre structure itself; (2) the extra stress applied to the structure due to thermal expansion effect of UV glue and alcohol; (3) some approximations in the simulation module as mentioned earlier in this section.

To further demonstrate the improved performance for temperature sensing due to the filled alcohol, a temperature sensing comparison experiment based on an SNCS fibre structure with the identical optical fibres but without surrounding alcohol was performed. The green line shown in Fig. 7 is the fitting result of the measured amplitude change (in $\mathrm{dB}$ ) at a wavelength of $1531.8 \mathrm{~nm}$ (which is the central wavelength of dip $_{1}^{\prime}$ in Fig. 4) over the temperature range of $20^{\circ} \mathrm{C}$ to $45^{\circ} \mathrm{C}$. From Fig. 7, it is clear that the achieved temperature sensitivity of SNCS fibre structure surrounded with alcohol is more than 300 times higher than that $0.0015 \mathrm{~dB} /{ }^{\circ} \mathrm{C}$ of the SNCS fibre structure without alcohol, which is due to the high thermos-optic coefficient of alcohol. Based on the above results, a novel temperature sensor based on an SNCS fibre structure combined with alcohol by monitoring the amplitude (in $\mathrm{dB}$ ) change at a single wavelength proved to be feasible.

In order to characterize the sensor more completely, the repeatability and response time of the proposed temperature sensor have also been investigated. To demonstrate the repeatability of the proposed fibre-optic temperature sensor, the sensor sample underwent

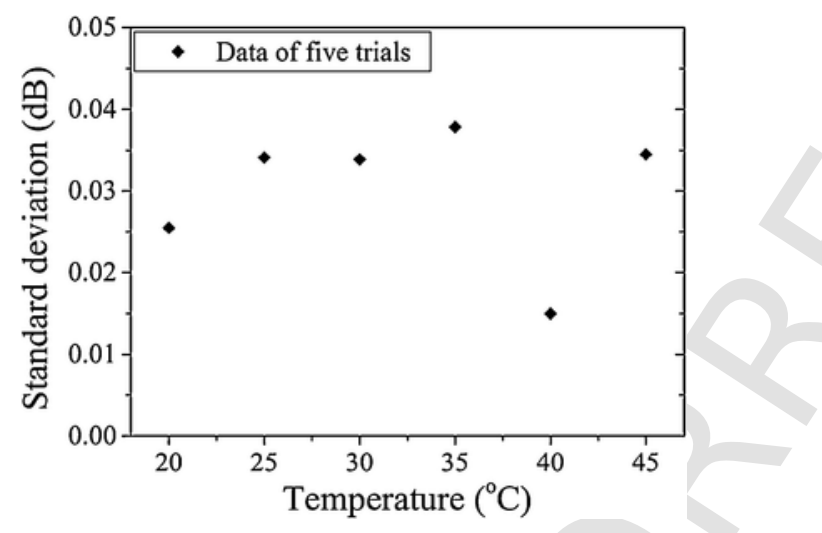

Fig. 8. Standard deviation of five trials

(a)

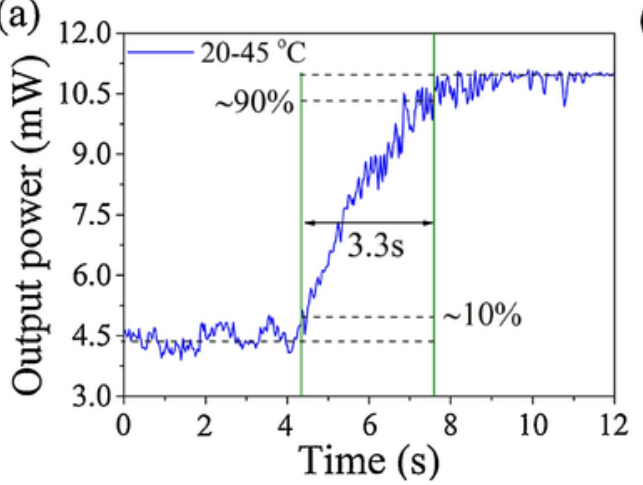

temperature cycling between $20^{\circ} \mathrm{C}$ to $45^{\circ} \mathrm{C}$ which was repeated five times. The standard deviation from the results of the five trials were calculated and the results are shown in Fig. 8. From the calculated results, the highest standard deviation is just $0.038 \mathrm{~dB}$, which demonstrates the excellent repeatability of the sensor of this investigation. It is worth noting that the repeatability is significantly affected by the infiltration process of the alcohol solution. For example, if during the immersion process the amount of the alcohol solution injected is not sufficient to fill the space available, this can result in air bubbles within alcohol in the capillary tube which is found to reduce the repeatability. In addition, the UV glue must provide a good seal to prevent leakage and evaporation of the alcohol which can also impact negatively on the performance of the proposed sensor.

In the case of liquid-assisted temperature sensors, it is also important to determine response time. A tunable laser (NetTEST), a photo-detector and an oscilloscope (KEYSIGHT MSO-X $2022 \mathrm{~A}$ ) were used to measure the response time of the sensor in this investigation. To examine the response time for increasing temperature, a step change in the temperature of the sensor structure is forced by abruptly attaching the sensor, previously at room temperature $\left(20^{\circ} \mathrm{C}\right)$, to the Peltier cooler with a set temperature of $45^{\circ} \mathrm{C}$. To examine the response to a decreasing temperature step, the sensor was abruptly removed from the Peltier cooler at $45^{\circ} \mathrm{C}$, in effect forcing a step decrease to the $20^{\circ} \mathrm{C}$ room temperature environment. When the tunable wavelength was set at $1545.9 \mathrm{~nm}$, the real-time output optical intensity variation was recorded using the oscilloscope. The $10 \%$ to $90 \%$ point method was used to calculate the response time [29]. Fig. 9(a) and (b) shows the calculated response times are $3.3 \mathrm{~s}$ and $5.4 \mathrm{~s}$, respectively, when the temperature was increased from $20^{\circ} \mathrm{C}$ to $45^{\circ} \mathrm{C}$ and decreased from $45^{\circ} \mathrm{C}$ to $20^{\circ} \mathrm{C}$. The measured response times are different in the case of temperature rise and drop scenarios. This can be attributed to the fact that an ideal and identical temperature step change, upward and downward are difficult to achieve in practice. The response time is limited by the thermal capacity of the sample formed by the alcohol's volume. Due to the small volume of alcohol used in the experiment (which was filled in the capillary tube), the response time of the proposed temperature sensor is within an acceptable range for many biomedical and environmental applications.

Experimental results have shown that this sensor can work over the temperature range of $20-45^{\circ} \mathrm{C}$ in a highly stable and repeatable manner. The detectable temperature range is limited by the effective working temperature of the UV gel as well as the large thermal expansion coefficient of alcohol. Therefore, this sensor structure can access a higher temperature range if a more appropriate packaging and sealing material is adopted. In addition, whereas many optical fibre tempera- (b)

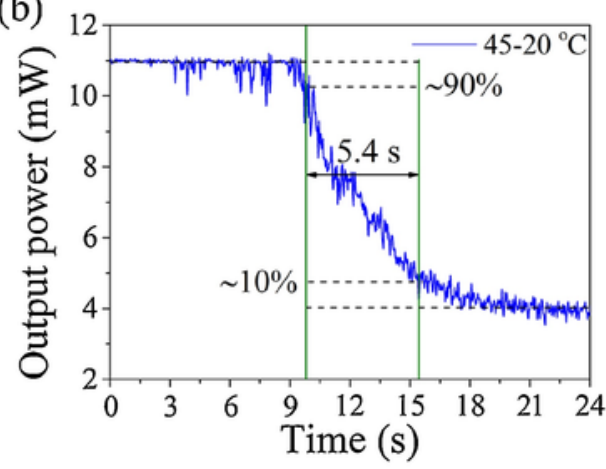

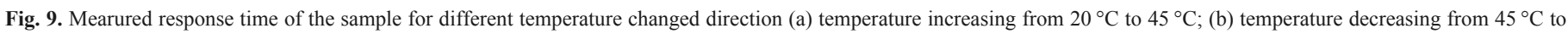
$20^{\circ} \mathrm{C}$. 
ture sensors' interrogation systems are based on wavelength shift, the temperature sensor in this investigation is based on intensity modulation. Thus, the OSA and the BBS which were used in the original experiment can be replaced by an optical power meter and a fixed wavelength LED or a laser light source with an appropriate choice of wavelength, which greatly reduces the overall cost of the measurement system.

\section{Conclusion}

A highly sensitive temperature sensor based on a singlemode-no-core-singlemode (SNCS) fibre structure in combination with alcohol has been described. In this investigation, no-core fibre (NCF) was used as the multimode waveguide and alcohol was chosen as the temperature sensitive medium acting effectively as a cladding. Through packaging alcohol with a short length of NCF within a sealed silica capillary, the surrounding temperature can be determined by monitoring the transmission loss at a specific wavelength. The experimental results show good agreement with the theoretical prediction, and a maximum temperature sensitivity of $0.49 \mathrm{~dB} /{ }^{\circ} \mathrm{C}$ has been achieved experimentally with a potential temperature resolution of $0.02{ }^{\circ} \mathrm{C}$ at the operating wavelength of $1545.9 \mathrm{~nm}$. Particularly, by monitoring the transmission loss change instead of wavelength shift, the cost of the temperature measurement system is significantly reduced. In addition, this temperature sensor has been demonstrated to have excellent repeatability and rapid response time, which confirms it can be potentially applied in different temperature monitoring scenarios including biomedical and environmental monitoring fields.

\section{Acknowledgements}

This work was supported by the National Natural Science Foundation of China (NSFC) (61575050); National Key R\&D Program of China (2016YFE0126500); Key Program for Natural Science Foundation of Heilongjiang Province of China (ZD2016012); the Open Fund of the State Key Laboratory on Integrated Optoelectronics (IOSKL2016KF03); China Postdoctoral Science Foundation (2016M601415); the 111 project to the Harbin Engineering University (B13015); the Government of Ireland International Scholarship programme; and the $\mathrm{Ph}$. D Student Research and Innovation Fund of the Fundamental Research Funds for the Central Universities (HEUGIP201820).

\section{References}

[1] Y. Zhao, L. Cai, X.-G. Li, F.-C. Meng, Liquid concentration measurement based on SMS fiber sensor with temperature compensation using an FBG, Sens. Actuators, B 196 (2014) 518-524.

[2] Q. Wu, Y. Semenova, B. Yan, Y. Ma, P. Wang, C. Yu, et al., Fiber refractometer based on a fiber Bragg grating and single-mode-multimode-single-mode fiber structure, Opt. Lett. 36 (2011) 2197-2199.

[3] T. Uematsu, Y. Ishizaka, Y. Kawaguchi, K. Saitoh, M. Koshiba, Design of a compact two-mode multi/demultiplexer consisting of multimode interference waveguides and a wavelength-insensitive phase shifter for mode-division multiplexing transmission, J. Lightwave Technol. 30 (2012) 2421-2426.

[4] Q. Wang, G. Farrell, Multimode-fiber-based edge filter for optical wavelength measurement application and its design, Microwave Opt. Technol. Lett. 48 (2006) 900-902.

[5] W.S. Mohammed, P.W. Smith, X. Gu, All-fiber multimode interference bandpass filter, Opt. Lett. 31 (2006) 2547-2549.
[6] H. Fukano, Y. Kushida, S. Taue, Sensitivity improvement of optical-fiber temperature sensor with solid cladding material based on multimode interference,", Jpn. J. Appl. Phys. 54 (2015) 032502.

[7] K. Tian, G. Farrell, X. Wang, W. Yang, Y. Xin, H. Liang, et al., Strain sensor based on gourd-shaped single-mode-multimode-single-mode hybrid optical fibre structure, Opt. Express 25 (2017) 18885-18896.

[8] K. Tian, Y. Xin, W. Yang, T. Geng, J. Ren, Y.-X. Fan, et al., A curvature sensor based on twisted single-mode-multimode-single-mode hybrid optical fiber structure, J. Lightwave Technol. 35 (2017) 1725-1731.

[9] X. Wang, G. Farrell, E. Lewis, K. Tian, L. Yuan, P. Wang, A humidity sensor based on a singlemode-side polished multimode-singlemode (SSPMS) optical fibre structure coated with gelatin, J. Lightwave Technol. 35 (2017) 4087-4094.

[10] P. Wang, G. Brambilla, M. Ding, Y. Semenova, Q. Wu, G. Farrell, High-sensitivity, evanescent field refractometric sensor based on a tapered, multimode fiber interference, Opt. Lett. 36 (2011) 2233-2235.

[11] Y. Chen, Q. Han, T. Liu, X. Lan, H. Xiao, Optical fiber magnetic field sensor based on single-mode-multimode-single-mode structure and magnetic fluid, Opt. Lett. 38 (2013) 3999-4001.

[12] H. Wang, S. Pu, N. Wang, S. Dong, J. Huang, Magnetic field sensing based on singlemode-multimode-singlemode fiber structures using magnetic fluids as cladding, Opt. Lett. 38 (2013) 3765-3768.

[13] C. Liao, Y. Wang, D. Wang, M. Yang, Fiber in-line Mach-Zehnder interferometer embedded in FBG for simultaneous refractive index and temperature measurement, IEEE Photonics Technol. Lett. 22 (2010) 1686-1688.

[14] V. Bhatia, A.M. Vengsarkar, Optical fiber long-period grating sensors, Opt. Lett. 21 (1996) 692-694.

[15] K. Tian, G. Farrell, E. Lewis, X. Wang, H. Liang, P. Wang, A high sensitivity temperature sensor based on balloon-shaped bent SMF structure with its original polymer coating, Meas. Sci. Technol. 29 (2018) 085104.

[16] H.Y. Choi, K.S. Park, S.J. Park, U.-C. Paek, B.H. Lee, E.S. Choi, Miniature fiber-optic high temperature sensor based on a hybrid structured Fabry-Perot interferometer, Opt. Lett. 33 (2008) 2455-2457.

[17] L.-Y. Shao, Y. Luo, Z. Zhang, X. Zou, B. Luo, W. Pan, et al., Sensitivity-enhanced temperature sensor with cascaded fiber optic sagnac interferometers based on Vernier-effect, Opt. Commun. 336 (2015) 73-76.

[18] E. Li, X. Wang, C. Zhang, Fiber-optic temperature sensor based on interference of selective higher-order modes, Appl. Phys. Lett. 89 (2006) 091119.

[19] P. Wang, M. Ding, L. Bo, C. Guan, Y. Semenova, Q. Wu, et al., Fiber-tip high-temperature sensor based on multimode interference, Opt. Lett. 38 (2013) 4617-4620.

[20] S.M. Tripathi, A. Kumar, R.K. Varshney, Y.B.P. Kumar, E. Marin, J.-P. Meunier, Strain and temperature sensing characteristics of single-mode-multimode-single-mode structures, J. Lightwave Technol. 27 (2009) 2348-2356.

[21] S. Silva, O. Frazão, J. Santos, F. Malcata, A reflective optical fiber refractometer based on multimode interference, Sens. Actuators B: Chem. 161 (2012) 88-92.

[22] A. Hatta, G. Rajan, Y. Semenova, G. Farrell, SMS fibre structure for temperature measurement using a simple intensity-based interrogation system, Electron. Lett 45 (2009) 1069-1071.

[23] A. Mufarrikha, A.M. Hatta, S. Koentjoro, SMS fiber structure with a multimode fiber graded index type for a temperature measurement using an intensity-based interrogation system, Int. Semin. Photon. Opt. Appl. (2015), pp. 944410-944410.

[24] Q. Wang, G. Farrell, W. Yan, Investigation on single-mode-multimode-single-mode fiber structure, J. Lightwave Technol. 26 (2008) 512-519.

[25] Q. Wu, Y. Semenova, P. Wang, G. Farrell, High sensitivity SMS fiber structure based refractometer-analysis and experiment, Opt. Express 19 (2011) 7937-7944.

[26] L.B. Soldano, E.C. Pennings, Optical multi-mode interference devices based on self-imaging: principles and applications, J. Lightwave Technol. 13 (1995) 615-627.

[27] E. Li, Temperature compensation of multimode-interference-based fiber devices, Opt. Lett. 32 (2007) 2064-2066.

[28] W. Qian, C.-L. Zhao, S. He, X. Dong, S. Zhang, Z. Zhang, et al., High-sensitivity temperature sensor based on an alcohol-filled photonic crystal fiber loop mirror, Opt. Lett. 36 (2011) 1548-1550.

[29] C. Bariáin, I.R. MatíAs, F.J. Arregui, M. López-Amo, Optical fiber humidity sensor based on a tapered fiber coated with agarose gel, Sens. Actuators B Chem. 69 (2000) 127-131. 


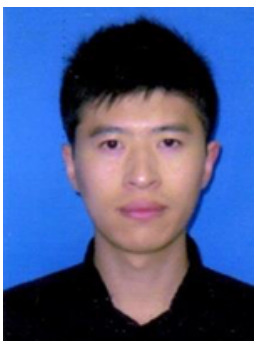

Ke Tian received the B.S. degree in physics from Heilongjiang University (HLJU), Harbin, China in 2014. From 2015 to now, he is an $\mathrm{MD}-\mathrm{PhD}$ student who pursuits for a doctorate in optical engineering at college of science, Harbin Engineering University (HEU), Harbin, China. From May 2017 to May 2018, he was awarded the Irish Government International Education Scholarship and visited to Photonics Research Centre (PRC), Dublin Institute of Technology (DIT), Dublin, Ireland. His research interests include fibre-optic sensors and devices.

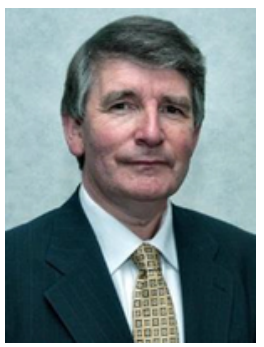

Gerald Farrell received his pH.D. degree from Trinity College Dublin for research in all-optical synchronization using self-pulsating laser diodes. Between 1997 and 2003 he was a director of the startup company PX Instrument Technology, focusing on optical fibre system test and measurement systems. Prof. Farrell is the founder and Director of the DIT Photonics Research Centre (PRC). He leads a multinational research team of doctoral, postdoctoral and senior researchers focusing on several areas of optical fibre sensing research including photonic crystal fibre sensors for environmental and energy systems sensing, novel sensors for composite materials and medical devices, LC infiltrated PCF sensors and micro-fiber and nanowire sensors for chemical and bio-sensing. Prof. Farrell has also led the development of research collaborations with a number of research groups in China, Poland and elsewhere. He has over 320 publications in the area of photonics. Prof. Farrell is also a member of the Optical Society of America and an advisory professor in China at Beijing University of Posts and Telecommunications and at Harbin Engineering University. He is also Dean of the College of Engineering and Built Environment at the DIT.

Xianfan Wang received the B.S. degree in applied mathematics from Harbin Engineering University, Harbin, China, in 2015. He received the Master's degree in Optical Engineering from Harbin Engineering University, Harbin, China, in 2018. His research interests include fibre-optic sensors and devices.
Yifan Xin received her B.S. degree of Communication Engineering at the Harbin Engineering University (HEU) in Jun. 2015. She began her career as a graduate student in College of Science, Harbin Engineering University in Aug. 2016. Her research interests include compound glass materials and fibre optic sensor. Her current research in focused on rare earth doped $\mathrm{SiO}_{2}$ fiber sensor.

Du Yanqiu received her pH.D. degree of physical electronics from Harbin Institute of Technology, instructor of Electronic and Informaiton Engineering College of Heilongjiang University of Science and Technology, engaged in postdoctoral research in Harbin Engineering University supervised by professor Wang Pengfei, introduced by the 1000 overseas talents plan of Organization Department of the CPC Central Committee, in the research field of Micro/nano optical fibre laser active devices on mid-infrared wavelength, and Micro/nano optical fibre laser sensors since 2016. So far, she has hosted one project of national postdoctoral science foundation, participated in four projects of Heilongjiang provincial natural science foundation, and published more than ten papers in the Sci-indexed journals such as Optics Letters and Optics Express. She is also the academic reviewer of the journals of Optics Letters and Optics Express for years.

Wenlei Yang received the pH.D. degree in photonics from Harbin Engineering University, China, in 2016. She is currently working toward the post-doctoral the Key Lab of In-fiber Integrated Optics, Ministry Education of China, Harbin Engineering University, China. Her research interests include fiber-optic sensing and long period fibre grating.

Haidong Liang received his B.S. and Master's degree in clinical medicine from Jilin University. He began his career as a $\mathrm{PhD}$ student in College of Science, Harbin Engineering University in Aug. 2016. His research interest is fibre optic sensor.

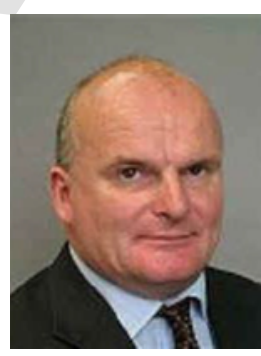

Elfed Lewis graduated with BEng (Hons) in Electrical and Electronic Engineering from Liverpool University in 1978 and was awarded his $\mathrm{PhD}$ from the same institution in 1987. He is Associate Professor and Director of the Optical Fibre Sensors Research Centre at University of Limerick, which he founded in 1996. He is Fellow of Institute of Physics, IET and Senior member IEEE. He has authored and co authored more than 70 journal papers and made in excess of 300 contributions to international conferences. He currently holds 7 patents on Optical Fibre Sensor Devices. In 2005 he was recipient of the University of Limerick Special Achievement in Research Award and was a Fulbright Scholar with CREOL (University of Central Florida) in 
2008. He was Distinguished Lecturer for IEEE Sensors Council for the period July 2013-June 2015 and General Chair of the recent European Workshop on Optical Fibre Sensors (EWOFS2016) held at University of Limerick, Ireland.

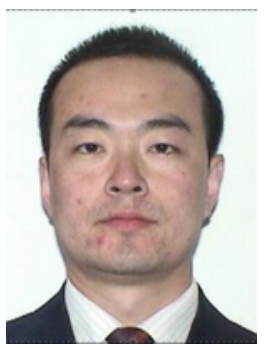

Pengfei Wang received his pH.D. degree of Optics Engineering at the Photonics Research Centre (PRC), Dublin Institute of Technology (DIT), Ireland in Nov. 2008. He worked for the European Space Agency and the Italian Space Agency as a Research Assistant at the Institute of Microelectronics and Microsystems, Italian National Research Council in Bologna Italy, sponsored by both the Italian Ministry of Foreign Affairs and the United Nations Educational, Scientific and Cultural Organization from 2004 to 2005 . He worked as a Re- search Associate in the Photonics Research Centre, Dublin Institute of Technology, Dublin Ireland, supported by the Irish Research Council Government Postdoctoral Scholarship (EMPOWER) from 2009 to 2010. In 2010, he joined the Optoelectronics Research Centre (ORC), University of Southampton, funded by the EU Marie Curie Research Fellowship. In 2011, he joined the Advanced Laser Lab of the SPI lasers (Southampton) as a research fellow. He has been appointed as a Senior Research Fellow (Tenured) at the PRC of the DIT from 2013 to 2015. From Sep. 2015, he has been working with the College of Science, Harbin Engineering University in China, as a distinguished professor, sponsored by the Chinese government programme entitled "The Recruitment Program of Global Youth Experts". His research interests include compound glass materials, fibre lasers, computational photonics (modelling, simulation and optimization), photonic devices (such as microfibre/nanowire based photonic devices, fibre optic sensors, photonic integrated circuits, liquid crystal based photonic devices, laser micro-machining etc) and applications development (optical communication and optical sensing). He has authored and co-authored over 200 papers in a number of academic journals and international conferences so far. 